\title{
PLATAFORMA DE NEgOCIO PARA EL FORTALECIMIENTO ECONÓMICO Y COMPETITIVO EN LA ASOCIACIÓN DE APICULTORES "AROMA\&MIEL"
}

\author{
AUTORES: Martha Irene Romero Castro ${ }^{1}$ \\ Rosario Magdalena Romero Castro ${ }^{2}$ \\ Marco Antonio Toala Pilay ${ }^{3}$ \\ Sornoza Pilay Jenniffer Estefanía ${ }^{4}$
}

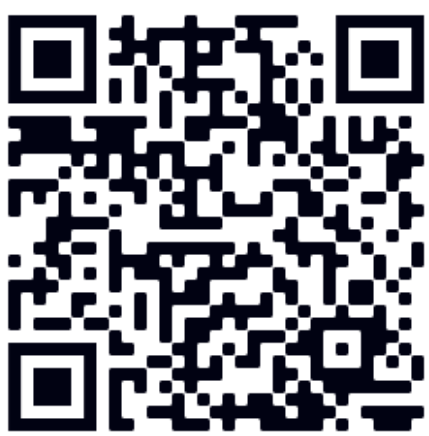

DIRECCIÓN PARA CORRESPONDENCIA:martha.romero@unesum.edu.ec

Fecha de recepción: 12/06/2019

Fecha de aceptación: 07/11/2019

\section{RESUMEN}

La presente investigación tuvo por objetivo la implementación de una plataforma de negocio por internet para el fortalecimiento económico y competitivo en la asociación de apicultores "Aroma \& Miel"en el recinto Quimis del cantón Jipijapa - Provincia de Manabí. La importancia de crear esta plataforma fue que constituyó una solución tecnológica, que permitió canalizar en forma efectiva y oportuna, la comercialización de los productos de los apicultores, aplicando técnicas de marketing apropiadas. La investigación está dividida en dos tipos: descriptiva, en la cual se aplicó un cuestionario a socios pertenecientes a la asociación de apicultores "Aroma \& Miel" Documental por el apoyo mediante medios electrónicos y bibliográficos. En la investigación se utilizaron métodos científicos como: análisis - síntesis, histórico - lógico, observación, encuestas y estadístico - matemático. Los resultados determinaron que la plataforma es una herramienta sofisticada y sustentable que ha permitido potenciar la factibilidad de este proyecto en el ámbito comercial y tecnológico, aportando de forma significativa al desarrollo económico, competitivo y emprendedor, con la demanda de ventas de productos apícolas. Se concluye que la implementación de la plataforma de negocios provocó un gran impacto tecnológico, económico, competitivo y emprendedor a todos los habitantes de la zona, beneficiando directamente a más de cien familias, se evidencia la mejora en su economía y calidad de vida de acuerdo a lo que menciona el plan nacional de buen vivir. Es recomendable, que los productores del sector implementen la plataforma para establecer el negocio a través del Internet.

PALABRAS CLAVE: competitividad, emprendimiento, organización, plataforma, productividad.

\section{BUSINESS PLATFORM FOR ECONOMIC AND COMPETITIVE STRENGTHENING IN THE ASSOCIATION OF BEEKEEPERS "AROMA \& MIEL"}

\footnotetext{
${ }^{1}$ Magister en Docencia Universitaria e Investigación Educativa, Magister en Informática Empresarial, Ingeniera en Sistemas. martha.romero@unesum.edu.ec

${ }^{2}$ Magister en Contabilidad y Auditoría, Ingeniera Comercial.

${ }^{3}$ Magister en Pedagogía de la Cultura Física, Licenciado en Cultura Física.
} 
Martha Irene Romero Castro, Rosario Magdalena Romero Castro, Marco Antonio Toala Pilay...

\section{ABSTRACT}

The objective of this research was the implementation of an internet business platform for economic and competitive strengthening in the beekeepers association "Aroma \& Miel" in the Quimis enclosure of the Jipijapa canton - Province of Manabí. The importance of creating this platform was that it constituted a technological solution, which allowed the marketing of beekeepers' products in an effective and timely manner, applying appropriate marketing techniques. The research is divided into two types: descriptive, in which a questionnaire was applied to partners belonging to the association of beekeepers "Aroma \& Honey" Documentary for support through electronic and bibliographic means. In the research, scientific methods were used such as: analysis - synthesis, historical - logical, observation, surveys and statistical - mathematical. The results determined that the platform is a sophisticated and sustainable tool that has allowed to enhance the feasibility of this project in the commercial and technological field, contributing significantly to economic, competitive and entrepreneurial development, with the demand for sales of bee products. It is concluded that the implementation of the business platform caused a great technological, economic, competitive and entrepreneurial impact to all the inhabitants of the area, directly benefiting more than one hundred families, the improvement in its economy and quality of life is evidenced to what the national plan of good living mentions. It is recommended that producers in the sector implement the platform to establish the business through the Internet.

KEYWORDS: competitiveness, entrepreneurship, organization, platform, productivity

\section{INTRODUCCIÓN}

Uno de los aspectos más relevantes en la actualidad es el uso de la tecnología tanto en el ámbito empresarial, educativo y social, y es por este motivo por el cual las pequeñas y medianas empresas hacen uso de los recursos que les facilita el internet y las computadoras para mejorar el proceso de interacción en usuarios y clientes. Esto con la finalidad de efectivizar y optimizar los procesos dentro de la organización.

El presente proyecto se ha desarrollado en respuesta a una necesidad comercial, administrativa y de gestión. Puesto que en la Asociación Aroma\&Miel se estaban presentando falencias en la distribución y comercialización de sus productos, demandando una gran preocupación tanto para los socios como los demás habitantes por el bajo nivel económico con el que ha venido existiendo en la zona desde hace varias décadas.

Cabe indicar que la producción y comercialización es unas de las actividades más sobresaliente de esta localidad rural, en cada época producción los habitantes invierten recursos económicos para la para obtener una cosecha significativa que a corto plazo necesita ser comercializada para generar ganancias y cubrir las inversiones, pero por la falta de marketing en muchas ocasiones no se suplen tales expectativas generando pérdidas irrecuperables.

Debido a esta gran necesidad nace la iniciativa de aportar con la comunidad y realizar una propuesta para el diseño e implementación de mejoras tecnológicas que permitan fortalecer económicamente a la asociación, mediante la potenciación de las actividades comerciales a través de un negocio por Internet. Utilizando una plataforma e-commerce o de comercio electrónico. 
En la actualidad este servicio ha crecido en gran demanda con el avance de las nuevas tecnologías existen una gran variedad de aplicaciones que permiten la utilización del procesamiento de datos automáticos a través de un software. Dentro de las funciones que ofrecen las plataformas de negocio por Internet, se tiene la facilidad de procesamiento de la información, disminución de gastos en material de oficina, fácil acceso a las redes y telecomunicaciones, contacto directo con el cliente, almacenamiento masivo y seguridad de la información.

El problema detectado en la Asociación "Aroma\&Miel” es el no contar con estrategias de negocios global, las cuales son indispensables para promover el desarrollo sostenible de las comunidades y más que todo de las familias de bajo recursos económicos, y sin lugar a dudas la tecnología permite proyectarse hacia los negocios por Internet en el cual se tiene un universo muy amplio de compradores.

Debido a estos aspectos se propone el diseño e implementación de una plataforma de negocio por Internet para el fortalecimiento económico y competitivo de la asociación de apicultores "Aroma\&Miel” del recinto Quimís del cantón Jipijapa, que permitirá innovar y mejorar las estrategias de comercialización de los productos que expenden e incluirlos en el mercado potencial para alcanzar una mejor rentabilidad económica del sector.

Los negocios hoy en día se han incrementado considerablemente y con el apoyo de la tecnología, muchos comerciantes o empresarios se dedican a implementar sus negocios en la web. Esto fomenta la investigación de mercados, donde se debe desarrollar nuevos productos, servicios o tendencias, en el cual la gente se involucre en el mercado tecnológico, satisfaciendo necesidades de los usuarios y su aplicación se vea fortalecida en portales o sitios web, para contribuir al desarrollo productivo y comercial. (Rocha, 2015).

Al usar una plataforma de comercio electrónico podrá vender en zonas geográficas, aceptar nuevos medios de pago y tener abierta su tienda virtual 24 horas al día los 365 días del año. Una tienda online es una efectiva forma de incrementar la distribución y evitar costosas inversiones relacionadas con la adecuación y administración de un punto de venta físico. (Gomez, 2017).

De acuerdo a (Martínez M. G., 2013), manifiesta que las estrategias y modelos de negocio de la prensa digital han sido objeto de estudio de una buena parte de académicos de la gestión mediática. El uso de terminología económica y del management ha provocado su utilización indiscriminada y cierta confusión entre ambos conceptos. La presente investigación contribuye a explicar y asentar términos sobre las estrategias y modelos de negocio desarrollados por la prensa digital. El objetivo es clarificar los términos y analizar los ejemplos de los diarios de referencia que los desarrollan.

Quedando demostrado que la aplicación del comercio electrónico tiene gran impacto a nivel comercial y de manera mundial, mejora notablemente los procesos de compra y venta, provee de catálogo para productos de alta calidad, facilidad de selección e incluso reducción de costos de operación y viáticos.

El comercio electrónico ha sufrido una expansión imparable desde el comienzo de la era de la información, convirtiéndose así en una de las actividades más relevantes de la economía nacional e internacional. Las principales causas para explicar dicho aumento, son el aumento del número de internautas y una mayor predisposición a la compra online. (Fernández \& Medina, 2018).

El manejo de información, la toma de decisiones y el uso intensivo de las TIC se hace cada día más presente en la vida laboral, personal y académica. Quien se encuentra en el ámbito de los negocios internacionales, cada día requiere de mayor información a fin de lograr los objetivos empresariales 
Martha Irene Romero Castro, Rosario Magdalena Romero Castro, Marco Antonio Toala Pilay...

que persiga. Se enfrenta ante una situación de estar en una continua toma de decisiones y de estar omnipresente en varias actividades cotidianas de distinto orden. (Martínez, 2017).

En la actualidad Internet no es un canal de comunicación que se deba subestimar y cada vez más empresas lo utilizan como parte integrante de su estrategia de marketing y publicidad. El ahorro de costes, una mayor audiencia y un mayor grado de interactividad con el cliente/visitante son sólo algunos de los aspectos que elevan a Internet en la actualidad. (Durango, 2015).

Estas nuevas reformas han contribuido de manera especial con "alternativas tecnológicas que han demandado positivamente el comercio a gran escala. A nivel mundial el comercio electrónico ha influido en la venta de bienes a través de países, demostrando gran capacidad de comercialización mejoramiento de la economía." (Rodríguez, 2012).

En una investigación desarrollada por, (Andrade, 2014), en la Universidad de San Andrés, se planteó un análisis sobre el comportamiento del comercio electrónico en la ciudad de Guayaquil, con el objetivo de crear una comparación del uso de e-commerce desde la llegada del internet. La problemática demostró que "al cierre del 2013, el 9\% de las transacciones de compras de los clientes fueron realizadas en internet, el 56\% de la población realizaron negociaciones en línea".

Este análisis permitió conocer el índice de beneficios electrónicos que desde entonces se propagó entre las empresas para mejorar las ventas de sus productos e incluirlos en un mercado potencial local, nacional e internacional. La comparación dio un resultado positivo superó las cifras estimadas de utilización del comercio electrónico entre la población de la ciudad de Guayaquil.

La inclusión de las plataformas e-commerce en el plano empresarial ha demostrado grandes ventajas tanto para el productor como para el consumidor, el estudio realizado por Cabrera permitió conocer que el comercio electrónico brinda mejores beneficios en productos, calidad de servicios y mejoras en las ganancias económicas, disminución de costo y tiempo en la realización de compras y venta.

En tal sentido, cabe destacar que la competitividad se refiere a la capacidad de las unidades productivas-empresa, sectores, regiones o naciones para hacer frente a sus competidores en los mercados nacionales e internacionales.

Sin embargo, se debe señalar que el núcleo de la competitividad se inicia en la empresa, la cual dirige sus objetivos hacia la generación de riqueza en términos de beneficios, reducción de costos, la innovación y la diferenciación. Al ser competitiva la empresa, esta influye en el sector industrial al cual pertenece, no obstante, para que un sector empresarial o industrial sea competitivo tiene que poseer ciertas condiciones que potencien sus capacidades y permitan su permanencia en el largo plazo. (Saiz, 2016).

Según expresa (Castellanos, 2013), los sectores empresariales están constituidos por un conjunto de empresas y la competitividad del mismo se mide por la capacidad que tiene para colocar exitosamente sus productos en el mercado internacional en condiciones de libre competencia. Los sectores pueden considerarse de forma amplia, incluyendo organizaciones educativas, de investigación y de servicios vinculados a las empresas.

Por otra parte, desde la perspectiva del concepto de competitividad sistémica, la cual se caracteriza y distingue por reconocer que para alcanzar un desarrollo industrial exitoso se requiere no solo una función de producción en el nivel micro, o de condiciones económicas estables en el nivel macro, sino también por la existencia de medidas del gobierno y organizaciones privadas de desarrollo 
orientadas a fortalecer la competitividad de las empresas (nivel meso). Además, la capacidad de vincular las políticas meso y macro está en función de un conjunto de estructuras políticas y económicas, de un conjunto de factores socioculturales y patrones básicos de organización (nivel meta). (Castellanos, 2013).

\section{DESARROLLO}

\section{Materiales y métodos}

El presente estudio alcanzo un tipo de investigación descriptiva y documenta. Descriptiva, porque se limitó a describir el fenómeno estudiado, esto es, la plataforma de negocio para el fortalecimiento económico y competitivo en la asociación de apicultores "Aroma\&Miel" del recinto Quimis del Cantón Jipijapa. A su vez, se aplicó un cuestionario de 10 preguntas a 100 socios pertenecientes a la asociación de apicultores. Documental porque se ha recurrido a información secundaria proveniente de medios bibliográficos, electrónicos y revistas de investigación, donde se ha recopilado conceptos y opiniones importantes de otros investigadores para fundamentar la investigación.

Análisis - síntesis: se utilizó para analizar la plataforma de negocio.

Histórico - lógico: se utilizó en el desarrollo de la investigación.

Observación: permitió investigar y conocer la información necesaria para el proceso de la investigación.

Encuestas: para analizar y determinar el estado actual.

Estadístico - matemático: se analizaron los datos estadísticos de los cuestionarios.

Resultados

Como resultado de la aplicación del cuestionario a los 100 socios de la asociación de apicultores "Aroma \& Miel" del recinto Quimis del cantón Jipijapa se verificó que:

En lo referente al método de comercialización que utilizan para vender los productos apícolas, se determinó que el $32 \%$ de la venta se realiza de productores a consumidor y el $68 \%$ de productores a comerciantes. Esto demuestra que el canal de venta más utilizado se realiza al por mayor. Pero es importante dar a conocer que, mejorando las estrategias de promoción y marketing, se mejoraran la adquisición de clientes potenciales que compren los productos apícolas a mejores precios, aumentando las ganancias de los asociados.

En cuanto a los productos apícolas que se cosechan en la comunidad, el 64\% corresponde a la producción de miel dulce, el $28 \%$ a la miel agria y el $8 \%$ a la miel real.

La frecuencia que realizan las cosechas de las colmenas durante el año, afirman el 100\% que solo es una vez al año.

El 85\% de los productores expenden sus productos en los mercados mayoristas, mientras que el $15 \%$ en canal directo al por menor. Por ello es importante que se hagan uso de una herramienta más sofisticada a través del uso de la tecnología y que les permita obtener mayor clientela para sus ventas.

El 100\% de socios están de acuerdo con la inclusión del comercio electrónico como canal de distribución para la asociación. Es importante destacar que los socios no tienen mayores 
Martha Irene Romero Castro, Rosario Magdalena Romero Castro, Marco Antonio Toala Pilay...

conocimientos sobre el uso de la tecnología, pero sería factible llevar a cabo una capacitación para que puedan utilizar herramientas tecnológicas para la comercialización de sus productos.

Finalmente, el 100\% de los encuestados, manifiestan la utilización de una plataforma de negocio, para ello se implementó una aplicación de comercio electrónico para la asociación de Apicultores "Aroma\&Miel", a través de la interfaz dinámica de OSCOMMERCE, con la finalidad de que el uso y diseño de esta plataforma permita fortalecer las ventas de la organización de manera ONLINE. Por lo tanto, se realizó una categorización de las necesidades encontradas en la asociación de apicultores "Aroma\&Miel” del recinto Quimís del cantón Jipijapa con la finalidad de determinar la necesidad de creación de un espacio web de comercio electrónico. Se estableció la funcionalidad del sistema en base a los requerimientos, detallados a continuación:

Para el alojamiento: adquisición del hosting y dominio.

Para la funcionalidad:

Módulos para la venta de productos apícolas tales como: Miel de Abeja y Palo Santo.

Detalle de los precios para compras al por mayor y menor

Datos directos con los socios expendedores

Datos de la asociación

Estructura y organización jerárquica

Portafolio y Galería de productos

Para el diseño:

Una plantilla que permita llegar a los clientes por medio de una interfaz dinámica e informativa que destaca los tipos de productos que tiene para la venta la asociación donde se cuenta con un módulo para las compras.

Un esquema personalizado de acuerdo a las funciones específicas de la aplicación frente al usuario.

Fig. 1: Diagrama lógico de la plataforma de Negocio por Internet

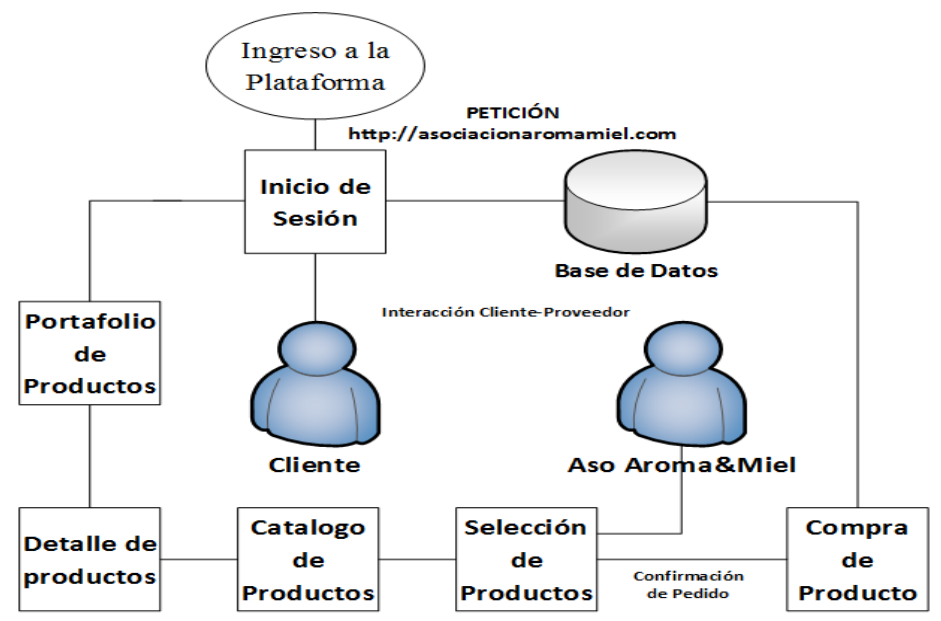

Para la implementación: 
Se implementó la plataforma web alojada en el dominio www.asociacionaromamiel.com, a los socios de la asociación, dirigiendo su uso a través de una previa capacitación al personal que estará a cargo del manejo.

Alojamiento en línea en el dominio www.asociacionaromamiel.com

Capacitación al personal de la Asociación de Apicultores “Aroma\&Miel” del recinto Quimís del cantón Jipijapa.

Fig. 2: Interfaz principal de la plataforma oscommerce

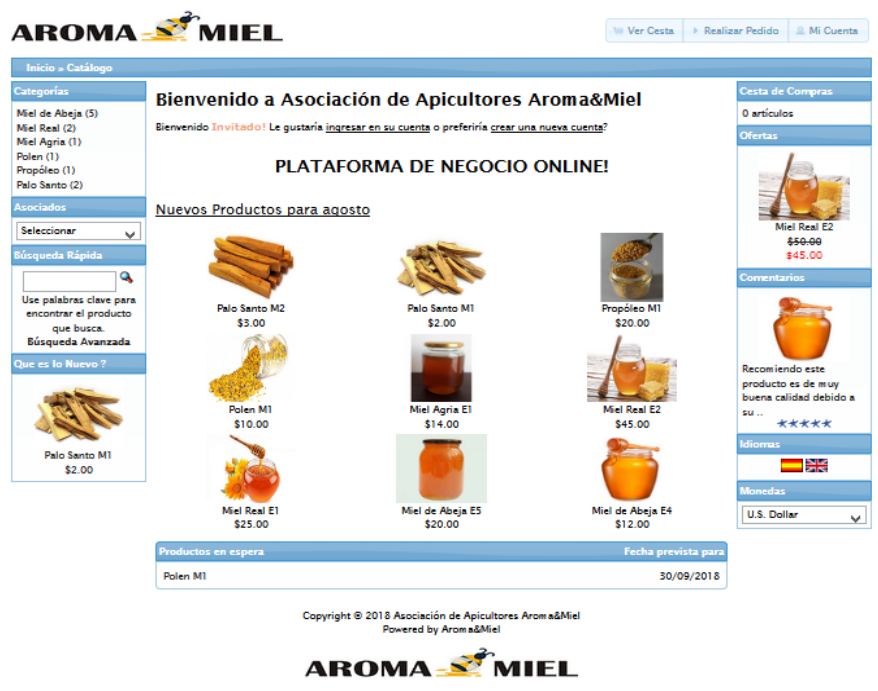

\section{CONCLUSIONES}

Una vez culminada la investigación se procede a platear las siguientes conclusiones:

Se verificaron los métodos de comercialización de productos apícolas en la Asociación de Apicultores "Aroma\&Miel". Los cuales son de venta directa ya que en la asociación no se cuenta con recursos tecnológicos para desarrollar otro tipo de marketing. Se lograron canales de distribución que utilizaron en la asociación para comercializar sus productos apícolas. El canal es directo y realiza las ventas de proveedor a vendedor. Se determinaron las técnicas de marketing más eficientes para mejorar la distribución de productos apícolas de la asociación, entre las que se estableció la utilización de una plataforma de comercio electrónico. La utilización de la plataforma ecommerce mediante osCommerce mejoró notablemente las estrategias de venta en la asociación de Apicultores "Aroma\&Miel”.

\section{REFERÉNCIAS BIBLIOGRÁFICAS}

Abbate, P. B. (2015). Ventajas del Marketing digital sobre el tradicional. Barcelona: LBC.

Andrade, C. L. (2014). E-COMMERCE EN ECUADOR: Estado actual y perpectivas de crecimiento. Villa Marin: Publi_Marin.

Cabrera, D. (2012). Analisis y aplicación de plataformas e-commerce en un portal web para pynes. Valencia: Valencia Editorial.

Castaño, J. J. (2016). Comercio electrónico. Editex., 14. 
Martha Irene Romero Castro, Rosario Magdalena Romero Castro, Marco Antonio Toala Pilay...

Castellanos Valle, J. A. (2013). Plan económico-financiero para fortalecer el desarrollo de las microempresas familiares dedicadas a la panificación en el municipio de Zaragoza. Doctoral dissertation, Universidad de El Salvador.

Comercio, O. M. (2015). Organización Mundial del Comercio. California.

Durango, A. (2015). La Guía Rápida de Comercio Electrónico. IT Campus Academy.

Fernández, M., \& Medina, J. (2018). El Comercio Electrónico. 40.

Gomez, D. G. (2017). El día que David venció a Goliat: Incremente la visibilidad de su negocio y atraiga más clientes, utilizando 6 palancas de marketing online. B DE BOOKS.

Guarrochena de Arjol, M. \&. (2013). Estrategias de gestión de la información asociadas a la inteligencia competitiva: apropiación práctica en organizaciones de apoyo a empresas exportadoras. Visión de futuro, 17.

Liberos, E. (2013). El libro del marketing interactivo y la publicidad digital. ESIC Editorial.

Llambí, P. (2014). Los seis modelos de negocio más rentable en Internet. Pastos: Columbia.

Martínez, M. G. (2013). Estrategias y modelos de negocio: aclaración de conceptos y terminología de la prensa en Internet/Strategies and Business Models: Clarification of Concepts and Terminology in the Digital. Press. Estudios sobre el mensaje periodístico, 13.

Martínez, R. M. (2017). Las tecnologías de información y comunicación: su evolución y aplicación en los negocios internacionales. Revista de Investigación en Ciencias de la Administración, 28.

Mundial, F. E. (2014). Reporte global de competitividad 2014-2015. Recuperado el, 21.

Muñiz, R. C. (2013). Marketing en el siglo XXI. Lima: Libros Piura.

Rice, E. A. (2018). El papel de la Ventaja Competitiva en el desarrollo económico de los países. Revista Análisis Económico, 28.

Rocha, A. (2015). Negocios en la Web, un Mall Virtual. Revista Científica y Tecnológica UPSE, 9.

Rodríguez, D. (2013). Negocios en Internet y algo más. 8.

Rodríguez, G. S. (2012). ELE-COMMERCE A NIVEL INTERNACIONAL. Madrid: Red Libros.

Saiz, V. I. (2016). El Enfoque de cadenas productivas y la planificación estratégica como herramientas para el desarrollo sostenible en Cuba. RIPS: Revista de Investigaciones Políticas y Sociológicas, 15.

Sepulveda Robles, D. (2014). Propuesta de modelo para Adopción del Comercio Electrónico en empresas del Sector Agroindustrial en México. 14.

Zurita, F. V. (2013). IMPACTO DEL COMERCIO ELECTRONICO EN EL ECUADOR. Cuenca: Bolivar. 